\title{
Data Management System for Ubiquitous Multi-Task Mobile Devices on Semi-Network OS Architecture
}

\author{
Yin Sheng Zhang
}

\begin{abstract}
This study is to explore a newly configured data management system of Internet of Things and other intelligent technologies in ubiquitous computing based on semi-network OS architecture.

Modern Internet of Things and other intelligent technologies have increasingly shown a trend of diversification and ubiquitous computing, and the usual mobile devices characterized by "single-task", "stable", "oriented", and "immutable" are also bound to face the pressure and motion of reform and innovation.

The advancement of merger of single-task mobile devices will be unstoppable, and the operating system rightfully needs to be divided into "base portion" and "expanded portion", which just fits the concept of semi-network operating system architecture; the "base portion" is similar to existing "embedded operating system", and the "expanded portion" is network resources.

Today, the merger of single-task mobile devices and the formation of multi-task mobile devices have become general trend, and architecture of semi-network operating system can lay a new foundation for this wave of merger.

The multi-task mobile device formed by merger of singletask mobile devices based on architecture of semi-network operating system has its special computing system: co-mobile computing system, where including a data management system.

Multi-task mobile devices form a so-called co-mobile computing system, and also form a newly configured secure data management system, which is like a universal key to form a new working system for opening a lot of unspecified locks based on new principle and security mechanism.
\end{abstract}

Index Terms - Architecture of semi-network OS, Comobile computing, Multi-task mobile devices, Single-task mobile device.

\section{INTRODUCTION}

This study is to explore a newly configured data management system of Internet of Things and other intelligent technologies in ubiquitous computing based on semi-network OS architecture.

Whether it is the Internet of Things or other intelligent technologies, they should require mobile devices to perform their tasks; the higher the depth of development and popularization of intelligent technologies, the more types of mobile devices are needed by people, which also greatly challenges people's ability to carry these increasing mobile devices in daily life.

In today's situation, most mobile devices are equipped with an embedded operating system, which usually performs a single and stable computing task to achieve oriented and immutable functions, but modern Internet of Things and

Published on August 29, 2020.

Yin Sheng Zhang, University of British Columbia, Canada.

(corresponding e-mail: cxo888@ ${ }^{\circledR}$ hotmail.com) other intelligent technologies have increasingly shown a trend of diversification and ubiquitous computing, then the usual mobile devices characterized by "single-task", "stable", "oriented", and "immutable" are also bound to face the pressure and motion of reform and innovation.

There are various mobile devices in practical applications, but some of their basic components are usually the same, such as keyboards, external storage devices, RAM, network connectors, etc., they need to be supported by the same operating system elements and application software comprising hardware drivers, these operating system elements and application software components can also be regarded as stable parts of mobile devices embedded in chips or other closed memories; in addition, different mobile devices have many different hardware components and software for different technical purposes, which need to be supported by different flexible operating system elements and application software.

If the advancement of merger of single-task mobile devices is unstoppable, the above two requirements for operating system elements, comprising some application software, will be more and more concentrated in a mobile device; the phenomenon of that increasing technical features by increasing the types of mobile devices disappears,

The technical features of computing device is realized by system software and application software; the capacity of a device is limited, and the mobile device has the characteristics of miniaturization, and its capacity is smaller than other devices, so it is impossible for them to increase the technical features of device by the method of stacking a large number of system software application software without limitation, so in response to the embarrassing situation in the merger of single-task mobile, one of the effective solutions is to divide the software in the device into "basic" and "flexible", and the "flexible" software should have the attributes of "temporary use" and "disappear after use".

The division of "basic" and "flexible" technical features (generated by software) appears in a device, then the operating system also rightfully needs to be divided into "base portion" and "expanded portion", as a result, the emergence of the concept of semi-network operating system architecture has become inevitable.

The "base portion" as the most ideal local element, it is similar to the existing "embedded operating system", the "expanded portion" of operating system as the most ideal supplementary element, it is from network resources, and therefore, the "portion division of operating system" coincides with the concept of architecture of semi-network operating system. 
Today, with the rapid development of the Internet of Things and other intelligent technologies, the merger of single-task mobile devices and the formation of multi-task mobile devices have become the general trend and people's desires, and the architecture of semi-network operating system can lay a new foundation for this wave of merger.

The multi-task mobile device formed by merger of singletask mobile devices based on architecture of semi-network operating system has its special computing system: comobile computing system, where including a data management system.

As an image metaphor: in the past, a single-task mobile device can only serve a specific matter, which is like an ordinary key can only open a specific lock, but now a multitask mobile device can serve a lot of matters, which is like a universal key can open a lot of locks; the service object of universal key can be increased or decreased at will, and the security means adopted by universal key can be updated at any time.

Multi-task mobile devices form a so-called co-mobile computing system under architecture of semi-network operating system, and also form a newly configured secure data management system, which is like a universal key to form a new working system for opening a lot of unspecified locks based on new working principle and security mechanism.

\section{UBIQUITOUS COMPUTING}

The concept of ubiquitous computing brought computing technology into a diversification stage and led to many devices that equipped with different functions to be combined into a single device but with multi-task features.

Modern smart phones, as an example, achieved a technological leap in multifunction of communication device that is because both computing technology and network technology have been incorporated into cell phone, which is equivalent to the devices of computer and traditional phone to be combined into mobile one, this multi-devices and multi-technologies combination gives users great convenience and gets users ardently sought after.

In current electronic age, a variety of electrical devices, power outlets and electrical wires can be seen nearly everywhere, people get benefits from electrical devices, but at the same time, also worrying about too many electrical items accumulating around their place.

Many electrical items in reality have similar functions and purposes that they serve to, which make some users wonder, why not combine those electrical devices with similar features or applications into one? Smart phone has achieved such a combination, hasn't it?

Unfortunately, since the emergence of smart phones, even though the concept of ubiquitous computing has been very widely accepted by academia and business field, the emergence of ubiquitous computing devices are not as exciting as people think, most people still just link the concept of ubiquitous computing together with mobile phone or similar, therefore, although the product of ubiquitous computing has appeared in different forms, such as conveyances and industrial equipment, but mobile phone is still only one of the most dazzling products in market, and it is still a dream for people looking forward to that multiple similar electrical devices are combined into one under the concept of ubiquitous computing and form a tide of consumption.

Since there is such an embarrassing situation, an alternative solution will be provided here, which is the ubiquitous multi-task mobile device equipped with a seminetwork OS.

Semi-network OS is a new exploration to change traditional configuration mode of operating system, and wherein the "semi-network" refers to that the most of system files (expanded portion) of full semi-network OS is stored on network server, and the network server is a storage place of system files rather than operating place, and these system files can only be downloaded to client platform to perform their computing tasks; while the rest of the system files (base portion) of full semi-network OS are stored in OSPU on client platform.

OSPU is the abbreviation of "operating system processing unit", which is the most important supporting hardware for architecture of semi-network OS, and a hardware component of computing device for guiding expanded portion of semi-network OS to be downloaded to client platform, and providing base portion of semi-network OS to client platform.

OSPU is constituted with a set of non-volatile ROM chip located on client platform.

The study of architecture of semi-network OS may open a new path to break down the bottleneck in development of ubiquitous multi-task mobile device, and it also has a strong feasibility.

\section{ARCHITECTURE OF SEMI-NETWORK OS AND OSPU}

The architecture of semi-network OS and its OSPU (a component of computing device) try to increase computing device's mobility and security, broaden the prospect of device miniaturization, save resources, reduce application cost, and push forward ubiquitous computing device to take new step in innovation.

OSPU is a key element to realize the concept of architecture of semi-network OS, and its role in promoting the development of ubiquitous computing is also very obvious, which is not only reflected in the structural transformation of existing mobile devices, but also in the improvement of data management systems.

The architecture of semi-network OS and its OSPU are specifically designed for network environment, which will not only be applied on computing device, but also be applied to other electrical devices, such as TV set, therefore, which meet the concept of ubiquitous computing, namely: countless computing and communicating devices that serve different purposes are to be connected in network, but the machine of computer itself disappeared from people sight.

Semi-network OS and related OSPU can play their largest and most comprehensive features only when network being connected, thus, the computing and generating of system data and user data surely to be inseparable from network, a large number of responsibilities that currently borne by client platform, or directly borne by user surely to be transferred to network server for processing, such as software installing, software faults repairing, and program updating. 
Once network truly become center of client platform, the advantage of server resources and server system regulatory function would much more exceed a single computing device, or a single user, from this, users are expected to be liberated, costs are reduced, system failure rate is expected to be fallen, and troubleshooting becomes easy, so that it help to promote the concept of ubiquitous computing.

Semi-network OS and related OSPU can resolve viruses and other issues that network brings to client platform, even, which can make network to be a security means to protect client platform, because data flow dominated by OSPU should be checked by server, and server is generally recognized to be more stringent security system than client platform, so it enhance the security of ubiquitous computing device.

OSPU is not only a boot component, location component, and security component for system data and other kinds of data that downloaded from server to client platform, but also a regulation component for regulating overall operation of computing device, which facilitates a large number of various types of data that originally stored in client platform to transfer to network server for storage, thus, the function of client platform has more room to be increased, the size of client platform also has more room to be reduced, so which will provide more opportunity for innovation of ubiquitous computing device.

With concept of architecture of semi-network OS and OSPU, client platform is no longer a place to free install software, and hard drive or similar device is no longer the carrying device of operating system, which makes viruses, hackers, phishing and so on lost their activity place, lost their attack target, and lost their value of existence, which especially save a lot of economic resources in production and maintenance of operating system and related devices, thus contributing to reduce the size of device, lower the price of device, and contributing to market promotion of ubiquitous computing device.

The architecture of semi-network OS will take network as center to put all the function of data storage, data extraction and other data processing together; in this architecture, some security measures will be closely integrated and complement each other in OSPU, these measures include the hardware code of OSPU, the user's personal identifiable information and other personalized data security setting.

Therefore, the device can not only achieve full functionality, but also obtain more opportunities to remove some of existing components and facilitate to reduce the size of whole device.

As an example, after the concept of ubiquitous computing becomes a reality, a large number of daily household appliances will be replaced by intelligent household appliances equipped with exclusive operating systems, exclusive application software and exclusive hardware configurations, then it is necessary to establish an computing hub to intelligently monitor and remotely control household appliances within the scope of a home, and the intelligent device that act as home monitor and remote control hub should have the ability to surpass or break through the barriers that existing in different types of computing devices, including operating system barriers, application software barriers, and hardware configuration barriers, that is, home monitor and remote control hub should be compatible with various types of terminal intelligent household appliances.

Generally speaking, the traditional model of computing devices is difficult to surpass or break through those barriers of software and hardware existing in different computing devices, so most of those devices are not suitable to undertake the important task of home monitor and remote control hub in ubiquitous computing case.

However, architecture of semi-network OS and OSPU provide a new way to establish this kind of home monitor and remote control hub for ubiquitous computing, in this case, the expanded portion of semi-network OS (derived from server) will surpass and break through existing "barrier" to provide flexible support for home monitor and remote control hub, and the base portion of semi-network OS (derived from OSPU) will surpass and break through existing "barrier" to provide stable support for home monitor and remote control hub.

\section{Co-Mobile COMPUTING}

Co-mobile computing is a data computing system specially designed for networked multiple-tasks mobile devices based on a semi-network OS architecture, and its application fields are mainly artificial intelligence and network remote control.

Under the semi-network OS architecture, mobile devices with different embedded OS and functions are expected to be combined into one device, thus forms a co-mobile data computing system, therefore, one of the important prerequisites for realizing this co-mobile computing system is that a new concept of semi-network OS architecture to be applied to mobile devices.

The embedded OS adopts enclosed software installation mode, its hardware carrier forbids any other software installed freely on it (chips), and therefore, it has long been mainly used in mobile devices with single task with stable and unchangeable functions.

Nowadays, the concept of co-mobile computing requires the tasks of mobile devices transformed from simplification to diversification, and a feasible way is:

The base portion of OS in semi-network OS architecture serves as the embedded OS, and the expanded portion of OS in semi-network OS architecture is used to make the functions of embedded OS flexibly extended.

Therefore, the networked mobile devices can not only preserve the advantages of usual embedded OS, such as high security and strong user autonomy, but also overcome the shortcomings of usual embedded OS in functional flexibility.

Thus, although the co-mobile computing system relies on multiple innovative concepts, the most decisive one is the concept of semi-network OS architecture.

In co-mobile computing system, the base portion of OS (similar to usual embedded OS) in mobile devices should have the scalability and flexibility in its function, in order to facilitate the usual single-task mobile devices to be improved into a new pattern of multi-task one.

The important requirement above has been met by the expanded portion of OS under semi-network OS architecture, and the focus here is another important requirement of co-mobile computing system: the specificity 
of mobile devices and the exclusivity of data management system in co-mobile computing.

The co-mobile computing device can achieve the features of multi-tasking, but it is still a dedicated device, and it is not allowed other devices or other platforms without specified OSPU to access its exclusive data management system by other ways and means, even if these devices have the same verification credentials except for the specific OSPU, furthermore, it does not allow users to upload any data from other computing devices without specified OSPU to its exclusive data management system, or download any data from its exclusive data management system to other computing devices without specified OSPU.

In co-mobile computing system, the "exclusivity" requirement of data management system must be strictly enforced with no relaxation or negligence, because once this "exclusivity" is destroyed, for example, its data management system can be accessed by unexpected visitors from thirdparty platforms, which is like opening a convenient door for virus attacks, then co-mobile devices may be plunged into a disaster, which can be imagined from the consequences of virus harassment in the Internet of Things.

Under architecture of semi-network OS, the OSPU is the only access path for data management system in co-mobile computing, and its specific hardware identity and operation mechanism will strengthen its exclusive status, and if other security measures, such as "assembly verification", are combined to be applied, then, the "specificity" of mobile devices and the "exclusivity" of data management system can be more ideally realized, that is to say, the requirements of co-mobile computing system can be met.

\section{WEAKNess Of USUAL Data MANAGEMENT System}

The data management system is a very important supporting element to data computing of mobile devices, but some weakness of usual data management system are always difficult to obtain a very effective solution to be solved so far, which is especially reflected in following points:

A. There is a data management system on remote server, and there is another data management system on client platform, these two data management systems can only establish indirect contact through intermediary application software, and they cannot directly contact each other.

While data transmitted between client platform and remote server, it becomes a tedious technological practice and complicated issues of how to ensure the successful transfer of data across different networks and client platforms, or how to ensure the implementation of data processing can be performed synchronously and in the same standard on different platforms at both ends of network at all time.

Therefore, in the current practice, the endless stream of special software has to be installed on client platform to act as a contact intermediary to coordinate the data exchange relationship between platforms at both ends of the network, for example, some file format conversion software installed on local mobile device to ensure smooth data exchange between local mobile device and network server.

B. There is an independent so-called cloud OS on server, and there is another independent embedded OS on client platform, these two independent OS developed according to the requirements of their respective platforms and not related to each other.

Therefore, a special software application installed on client platform should serve as a bridge, to ensure critical data's consistency between server and client platform.

For example, a small size DB2 to be developed to support standard SQL API and SQL subset, so that users can transfer appropriate application into each client platform.

Alternatively, users can use same standard developsoftware and API to write new applications and to install these applications on each client platform in order to make these devices able to use data replication function and obtain data from database on server.

C. There is an independent security verification system on server, and there is another independent security verification system on client platform, and these two independent systems lack of the same standard.

One is on lower level but other is on higher level, so that server always difficult to get rid of the threat posed by malicious data from client platform during network transmission.

In particular, the same data management system, especially its subordinate account system can be accessed by multiple different client platforms with the same verification credentials, while the verification system has no absolutely effective measures to prevent the risk of forgery, counterfeiting, imitation and other malicious attacks for this situation.

All of the above issues are caused by the old configuration mode of usual data management system, therefore, it is very difficult to be solved without configuration mode innovation, now, above issues are expected to be solved under semi-network OS architecture.

In semi-network OS architecture, the system files need to be divided into two portions for different purposes, but these two portions belong to same OS, and need to be integrated into a completed one to fully operate on client platform.

Therefore, this "portion" division makes the activities of a variety of hardware and software that connected to network under the same OS's monitoring and management without extra special application software as bridge, and which omits the steps of data replication and software installation, then critical data will be always in consistency between server and client platform.

If usual embedded OS is sufficient for ordinary mobile device with single-task and simple function in the past, but now, in face of the trend toward multi-tasking and multifunctionality of mobile devices, namely, faced with ubiquitous computing, faced with the emergence of a large number of multiple-tasks mobile devices, it has become increasingly difficult to cope with.

As for the security verification, specific OSPU of seminetwork OS architecture will become the only path to access data management system in co-mobile computing, and other paths will be prohibited completely, in this case, even an OSPU is true, but it is not the specified OSPU, it will also be prohibited in a specified data management system access process. 


\section{SOLUTIONS}

The data management system for co-mobile computing requires the persistent data management mechanism, scalable data operating environment, and the ability to respond to large data flow from a variety of mobile devices.

In current situation, each mobile device has its standalone data management system, all system data and application data are in the form of software installed in external memory of mobile device, and various other data generated by mobile device is mainly stored on client platform for its single-task or for security purposes.

Now in co-mobile computing, after different types of functions integrated into one device, the amount of data in a single data management system may increase in multiples.

Co-mobile computing belongs to the category of interaction of server and local mobile devices, as a computing system that transforms the mobile devices with single and targeted computing tasks into multiple and comprehensive computing task, the data generated by local mobile devices inevitably to be affected or controlled by server to some extent.

Server acts as a supplementary source and adjusting means for data on local mobile devices, meanwhile, the data on server-side and local-side should be getting together and work collaboratively to achieve the desired results, so the data exchange between client platform and server becomes normal.

However, due to constraints of usual model of data management system, the data management system at serverside and local-side cannot be combined into a unified one, and they need the help of special application software to communicate across network effectively and securely; in addition, there is always a concerning matter during data exchange that how to ensure consistency of critical data.

A mobile device (mainly for artificial intelligence or remote control) may run more than one set of system and application data, in some particular cases, devices may even run user data.

All the running data stored in device not only affect the convenient-oriented characteristics of mobile device, but also increasing computing work load that eventually brings impact on system performance, squeezing device's capacity, and even may lead to data irretrievably lost due to damage of device's hardware components.

Obviously, the most efficient and convenient way is that: the system data and application data centralized stored in an unified server-side data management system ready to be download, and the number and variety of data download will be timely and intelligently adjusted by system according to actual needs of client platform.

Unfortunately, the usual data management system currently could not achieve this effect, and usual mobile device will always be difficult to get rid of the feature of lightweight computing, so the usual mobile device can only be used for certain specific applications.

\section{DATA CONSISTENCIES}

In existing pattern, the platforms at both ends of network have different data generation and processing mechanisms, and some of the data formats they create will also inevitably be very different, which require the data to be adjusted and modified by additional application software, so as to facilitate the smooth operation of the same data at both ends of network.

The consistency of critical data at both ends of network also relates to the security settings of data management system.

The process of allowing critical data adjusted and modified by additional application software, which also poses a threat to the security of critical data and data management system.

Moreover, if the data of the different platforms on both sides of network cannot maintain consistency, it is impossible effectively to use the means of uniqueness of verification paths, exclusivity of verification credentials and realness of user identity to ensure security of data management system.

The consistency requirement of data management system mainly refers to the consistency of critical data at both ends of network.

In usual system architecture, after data that generated on client platform is uploaded to server side, it will be verified and reprocessed based on another OS (cloud operating system), then the data will be transferred to server-side data base; data download is also with the same process; in this process, it is necessary to add additional operational links and application software to adjust the data type and format, etc. to ensure the consistency of critical data at both ends of network, which is a cumbersome and security risky process.

In contrast, semi-network OS architecture is based on local computing, all data are generated on client platform and uploaded to server with strict security verification, it will not be reprocessed based on another OS (cloud operating system) on server side, moreover, the data that directly generated and processed on server side based on cloud OS cannot be stored in data management system prescribed by semi-network OS architecture, so which can ensure the data consistency on different platforms at both end of network.

Semi-network OS architecture involves client platform and server, which are located at both ends of network, so which forms two-stage verification, first is security verification of client platform, and second is the security verification of server side, but as a security verification under the unified OS architecture, it adopts unified rules or standards of file format, data association, and security system design, in addition, it simplifies many tedious procedures and processes, and excludes the participation of other OS and related application software, so it is very different from the usual two-stage verification of data that usually done on the basis of local OS and cloud OS respectively.

\section{EXClusivity Of DATA MANAGEMENT System}

High exclusivity is an important feature of data management system in co-mobile computing.

Under semi-network OS architecture, only when OSPU is used to mobile device, user can access his personal data management system in co-mobile computing, and once an OSPU is inserted into a mobile device, even though the device has always been in a software free installation mode, 
the original usual OS in original device will no longer work, and its original device's hard disk will lost its original function; in this case, because semi-network OS runs in the RAM of device, and only exists temporarily in this RAM, therefore, those uninvited harmful software more or less existed in hard disks of original device will not work.

OSPU (Operating System Processing Unit) is an exclusive hardware component, and only the security verification credentials provided by OSPU are accepted by data management system in co-mobile computing, which including the " fragmented data " by assembly verification method; meanwhile, the access paths to data management system in co-mobile computing will be highly isolated when the hardware identity and other features of OSPU acts as verification credentials, in this case, the data management system in co-mobile computing cannot be accessible in any way under usual traditional system architecture.

In addition, the "data splitting and assembling" principle can be applied to the access verification of data management system, but under usual system architecture, because the new verification credentials that generated by a new method is beyond the scope of common file format that server can identify, so the new verification credentials is usually not acceptable, or is strictly restricted, or is repeatedly checked by server that equipped with usual system architecture.

However, "assembling verification" method is one of the security measures originally set up for semi-network OS architecture, which complements the OSPU security measures, therefore, it has no operational obstacles in seminetwork OS architecture.

The above-mentioned "assembly verification" method means that a complete file is divided into two irregular parts by special software and hardware devices on client platform or server platform, and forming the fragmented data that loses information value, then one of them is permanently stored on client platform and is never allowed to be stored on server side, which is called local fragmented data, while the other part is permanently stored on server and never allowed to be stored on client platform, which is called remote fragmented data, where "fragmented data" is a "data split file".

When the network security verification process is activated, the local fragmented data is sent from client platform to server through network path by user, and assembled with the corresponding remote fragmented data on server side, then if the file is successfully assembled, the network security verification will be successful, on the contrary, if the file assembly fails, the network security verification fails.

All in all, the "assembly verification" method and the security features of OSPU can effectively cooperate in seminetwork OS architecture, and which is a cooperation between two highly exclusive security measures that never seen under usual system architectures.

\section{SEMI-MOBILE ATTRIBUTES}

In this study, the data management systems are divided into two types in attribute: full mobile attributes and semimobile attributes, the purpose of this division is to better illustrate the difference between of data management system in co-mobile computing and usual one in traditional computing mode.

\section{A. Full-mobile attributes}

Here, all of the data management system involved in usual mobile devices are defined as full-mobile one, and the reason is that usual data management system in traditional computing mode are all regard specific mobile devices as data communication objects and data application destinations; as far as local data management system are concerned, the data management system are inseparable components of specific mobile devices and permanently follow and work together with specific mobile devices; as far as cloud data management system are concerned, the data downloads and uploads from or to data management system are directed and positioned by specific mobile devices, and all the data management system have been using specific mobile devices as computing platforms, especially, the OS data with user personalized settings in these data management system have to permanently follow specific mobile devices to work and cannot be transferred from one mobile device to another.

\section{B. Semi-mobile attributes}

Data management system with semi-mobile attributes belongs to the concept associated with co-mobile computing, which is for multiple-tasks mobile devices, essentially, the semi-mobile attribute of data management system in co-mobile computing is determined by the semimobile attribute of OSPU (Operating System Processing Unit).

Under architecture of semi-network OS, OSPU is only a component of computing device, but it has ability to take the OS with user personalized settings from one device to another by user's autonomous operation, as a result, users bring OSPU with them just as they bring their personal computing devices, so it is called the semi-mobile attribute of OSPU; in this case, the data management system in comobile computing will use OSPU (a component) as data downloading object, destination, and orientation marker instead of a complete set of mobile device, then to generate semi-mobile attributes of data management system in comobile computing.

As can be seen from the above, the full-mobile attributes is a concept based on a complete set of mobile device, and the semi-mobile attributes is a concept based on OSPU (a component of semi- mobile device); among them, the data management system with semi-mobile attributes in comobile computing is only applicable to architecture of semi-network OS, and it is especially suitable for networked multi-task mobile devices, because OSPU not only contains base portion of OS, which similar to usual embedded OS, but also provides flow channels, adjust gates and security checkpoints for expanded portion of OS, which is network resources from server, furthermore, OSPU has the ability to integrate the two different kinds of resources of OS into one and reprocess the integrated resources of OS on client platform for best system running on mobile devices.

As long as OSPU is used in specific mobile device, the data management system with semi-mobile attributes will be automatically activated by system, and according to the 
definition of co-mobile computing, the OSPU dominated mobile device will naturally become a member of co-mobile computing system.

\section{Discussion}

In traditional mode, a large amount of system software and application software must be permanently installed on a local device to ensure that the device's features are implemented smoothly and safely, among them, in particular, security software usually takes up most of storage space of local device.

Much software installed in local device is not for providing device with operating functions required by user, and they have only one purpose, that is, to coordinate the relationship of local devices and network servers, including the application software installed in local device for supporting cloud computing and for anti-network virus.

New software emerges in an endless stream, and in most cases, the old software that has been abandoned is hard to be removed in time and completely from devices, therefore which puts more and more burdens on data management system, and furthermore, the capacity expansion effect of devices created by technological progress is offset by waves of software increases.

This situation also directly affects the merger of singletask mobile devices and the implementation of ubiquitous computing.

Taking intelligent household appliances as an example, it is hard to imagine that an intelligent household appliance that makes users worry all day will be sought after by users and have an excellent market prospects, such as: users not only have to bear the responsibility of preventing intelligent household appliances from being invaded by network viruses, but also always worry about the quality of security software of intelligent household appliances; not only have to bear the responsibility for correct operation of intelligent household appliances, but also always worry about abnormal or interrupting running of intelligent household appliances caused by software factors, and bring inconvenience to their family life; not only have to bear the responsibility of software maintenance, but also always worry about their lack of ability to maintain software.

The semi-network operating system architecture and comobile computing are expected to keep the software data in multi-task mobile devices to the minimum required by the device task, because it has the expanded portion of seminetwork OS and a unified server-side application software database, then most of software data in local mobile device as a network resource is temporary for specific tasks; in addition, the base portion of semi-network OS is stored in OSPU, which can be separated from mobile device together with the base portion of semi-network OS, thus to provide greater development space for the simplification and miniaturization of mobile devices.

More importantly, the semi-network operating system architecture and co-mobile computing can transfer many professional responsibilities of software maintenance from local device to network server, and freeing users from various worries, and making users of intelligent household appliances feel relieved in using.
However, the ubiquitous computing system and multitask devices formed by semi-network operating system architecture discussed here can only be realized by transforming the old model data management system, and also the newly configured data management system can only be based on co-mobile computing.

\section{CONCLUSIONS}

The conclusions of this study are as follows

\section{A. The semi-network OS architecture facilitates the elimination of "bottleneck" in implementation of ubiquitous computing}

In ubiquitous computing, multi-task mobile devices may appear everywhere in any form around people, so the categories of computing devices may no longer be so clear anymore, and since then people will start paying more attention to the role of device instead of the spotlight they have today on computing device, the ubiquitous multi-task mobile devices will greatly improve their work efficiency, will provide more and more services to people at any time, any place and in any way without involvement of human, and will make people's work, study and life much easier than before.

If the concept of ubiquitous multi-task mobile device becomes an impetus to form a wave of merger of single-task mobile devices, it is bound to further offer new prospects for user to accept the concept of ubiquitous computing.

However, first of all, it have to remove the bottlenecks that hinder the progress of ubiquitous computing device, especially for ubiquitous multi-task mobile device, then make the effectiveness of ubiquitous computing device more widely recognized by people, but in existing technical mode, this action to remove the bottleneck is struggling to move forward.

Now, the semi-network OS can provide a productive option for the action to remove bottlenecks in progress of ubiquitous computing device.

The concept of semi-network OS applied to a variety of ubiquitous computing device not only can allow intelligent devices to develop in a smoother new way, but also can make many single-task mobile devices with different features into the wave of "combination", and form a new comobile computing system.

B. Co-mobile computing can make the multi-task mobile devices more in line with the requirements of ubiquitous computing

To put it simply, co-mobile computing is just a kind of mobile computing that based on architecture of seminetwork operating system, and it is applied to multi-task mobile devices formed by merger of single- task devices.

The multi-task mobile device is different from usual mobile devices in that:

Firstly, the multi-task mobile device has the base portion of operating system to ensure the stability and safety of device operation, so that it can retain the characteristics and advantages of usual mobile devices with embedded operating systems, wherein the base portion of OS is similar to the usual embedded operating system. 
Secondly, the multi-task mobile device has expanded portion of operating system as a supplement to the base portion of operating system, because the expanded portion of operating system comes from network shared resources, so the function of the mobile device presents the flexibility to expand or retract, and which provide technical support for merger of single-task mobile devices.

Thirdly, the multi-task mobile device has a detachable hardware component OSPU, which is a carrier for the base portion of operating system and a guide for the expanded portion of operating system, so that its data management system in co-mobile computing obtains semi-mobile attributes.

This semi-mobile attributes of data management system in co-mobile computing makes mobile devices to be freely separated and combined with the functions they carried, that is: the "function" of devices can be transferred from one "device" to another following OSPU, and makes mobile devices "mobile plus co-mobile" and becomes more "mobile".

C. Ubiquitous computing based on semi-network OS architecture requires the support of a newly configured data management system

The data management system in co-mobile computing is a more secure system than usual for the reasons: the OSPU is the key credential for network verification and the exclusive channel for system access in this data management system.

The data management system in co-mobile computing is a more lightweight computing system than usual, that is because: the architecture of semi-network operating system is based on local computing, and there is no requirement for server computing, in addition, under a unified operating system architecture involving resources at both ends of the network, there is no special application software is needed to solve the problem of platform coordination at both ends of the network, which simplifies the content and processes of data management system, thus facilitates the creation of lightweight mobile devices.

The data management system in co-mobile computing is newly configured, which also greatly improves the safety and convenience of mobile devices, the reasons of "more convenient" are: the merger of mobile devices not only facilitates the streamlining of operation of data management system, but also facilitates the centralization and unification of data management system, which is beneficial to reduce the repeated occupation and inefficient consumption of resources of data management systems by mobile devices, and to reduce friction in application of data management systems among multiple mobile devices.

In short, the concept of ubiquitous computing rely on computing device to implement, computing device rely on operating system and application software to be able to operate, and operating system and application software are inseparable from the support of data management system, the optimal operation of this logical dependence relationship is exactly what the semi-network operating system architecture and its extended concept: co-mobile computing pursue.

\section{REFERENCES}

[1] Banavar, G. \& Bernstein, A. 2002. Software Infrastructure and Design Challenges for Ubiquitous Computing Applications. CACM, Dec. 2002/Vol. 45, No. 12.

[2] Demers, A.J. 1994. Research issues in ubiquitous computing. In: Proceedings of the 13th Annual ACM Symposium on Principles of Distributed Computing. ACM. New York, NY. USA.

[3] Lyytinen, K. \& Yoo, Y. 2002. Issues and Challenges in Ubiquitous Computing. Communication of the ACM, Vol. 45, No. 12.

[4] Chervenak, A., Vellanki, V., \& Kurmas, Z. (1998). Protecting file systems: A survey of backup techniques. Paper presented at the Joint NASA and IEEE Mass Storage Conference.

[5] Geer, D. (2008). Reducing the storage burden via data deduplication. Computer, 41(12), 15-17.

[6] Nakamura, S., Nakayama, K., \& Nakagawa, T. (2009). Optimal backup interval of database by incremental backup.

[7] Policroniades, C., \& Pratt,I. (2004). Alternatives for Detecting Redundancy in Storage Systems Data.Paper presented at the USENIX Annual Technical Conference, General Track.

[8] Reed, D. A., Gannon, D. B., \& Larus, J. R. (2011). Imagining the future: Thoughts on computing. Computer (1), 25-30.

[9] Renuga, K., Tan, S., Zhu, Y., Low,T., \& Wang, Y. (2009). Balanced and efficient data placement and replication.

[10] Jianli Pan, Raj Jain, Subharthi Paul, and Chakchai So-In. Milsa: A new evolutionary architecture for scalability, mobility, and multihoming in the future internet. Selected Areas in Communications, IEEE Journal https://www.cse.wustl.edu/ jain/papers/ftp/milsajsc.pdf.

[11] Zhang, Y. S. (2019). Applicability analysis of semi-network operating system. Computer and Information Science, 12(1), 93-97. doi: $10.5539 / \mathrm{cis}$. v12n1p93.

[12] Zhang, Y. S. (2019). Semi-network OS and new mode of application software sharing.Journal of Multidisciplinary Engineering Science and Technology (JMEST), 6(5), 10083-10088. Retrieved from http://www.jmest.org/wp-content/uploads/JMESTN42352949.pdf.

[13] Zhang, Y. S. (2019). Semi-network OS and Embedded OS for Comobile Computing. International Journal of Computing Sciences Research, Vol. 3, No. 2, pp. 189-198. doi: 10.25147/ijcsr.2017.001.1.32. Retrieved from https://www.stepacademic.net/. 